\title{
Screening for Mild Cognitive Impairment: There Is the Will but Is There a Way?
}

\author{
J.E. Galvin \\ Miami, USA, E-Mail: jeg200@miami.edu \\ J Prev Alz Dis 2020;3(7):144-145 \\ Published online April 6, 2020, http://dx.doi.org/10.14283/jpad.2020.16
}

Correspondance author: James E. Galvin, MD, MPH, Comprehensive Center for Brain Health, Department of Neurology, University of Miami Miller School of Medicine,

A lzheimer's disease (AD) currently affect over 5.8 million Americans and over 35 million people worldwide (1). The number of AD cases is expected to increase as the number of people over age 65 grow by $62 \%$ and the number over age 85 is expected to grow by $84 \%(1,2)$. More than one in eight adults over age 65 has dementia, and current projections indicate a three-fold increase by 2050 . Thus, the prevalence, incidence, morbidity, and mortality for $\mathrm{AD}$ (3) and its prodromal state of Mild Cognitive Impairment due to AD (MCI-AD) (4) will increase dramatically and the societal financial burden of illness and dependency will expand exponentially. Primary care providers are often responsible for the detection, diagnosis, and treatment of $\mathrm{AD}$ as the number of dementia specialists (neurologists, psychiatrists, and geriatricians) and specialty centers is not sufficient to meet the growing demands $(2,5)$. The inability to detect MCI and ADRD may affect eligibility determination for care and services and impede case ascertainment and recruitment in clinical research.

Despite these obvious demands for early detection, MCI-AD is often under-recognized in community practice, with many individuals obtaining a diagnosis in the mild-to-moderate stages of dementia, particularly in older adults from underrepresented and underserved communities $(2,5)$. MCI-AD screening would increase case identification; however, there are questions as to whether increased screening and case identification have value in the absence of more effective interventions that can improve patient outcomes (6).

In recognition of current challenges around the detection of MCI-AD, an international expert working group was convened in April 2019 to describe the opportunities and challenges to make a diagnosis of MCI$\mathrm{AD}$ in the clinic and home settings. This conference led to a series of three papers in the current issue of Journal of Prevention of Alzheimer's Disease (7-9). The first paper discusses pros and cons of early detection based on current research and considers how the changing landscape of technology and scientific advances will alter these perceptions (7). The second paper discussing methods for MCI-AD screening in the primary care setting, where most patients are encountered (8). The third paper offers thoughts on how MCI-AD screening could potentially be done in the home setting, with reports being made available to primary care providers at office visits (9).

MCI-AD screening is ideally suited for the primary care setting as the providers typically have long standing relationships with patients, have more readily available appointment slots, are already following other chronic conditions, and can provide continuity of care $(2,10)$. In particular, the Medicare Annual Wellness visit was designed to take advantage of this setting and has assessment of cognitive function as a requirement. However only $20 \%$ of Medicare beneficiaries have an Annual Wellness Visit and there are no clear guidelines as to what constitutes a cognitive assessment (1). In primary care settings, this is unlikely to include the detailed neuropsychological tests or invasive biomarkers often used in research settings or at University-based memory disorder centers.

A Special Report by the Alzheimer's Association "Alzheimer's Detection in the Primary Care Setting" (1) focused on early AD and MCI detection in the primary care setting (which constitutes $1 / 3$ of medical workforce in the US), where the majority of patients are seen and the first point of contact for most individuals. Particularly informative were the results of two surveys: (a) 1000 primary care providers and (b) 1954 older adults regarding expectations, benefits and practices about $\mathrm{AD}$ and MCI screening. While $94 \%$ of patients saw their primary care providers in the last year, only $47 \%$ discussed memory and $28 \%$ received a memory assessment. This contrasts with $95 \%$ of older adults wanting to know about their memory and 51\% reporting changes. Providers reported that $50 \%$ assess cognition as part of their evaluation but only $40 \%$ are familiar with the toolkits available to them. For those that do assess cognition, only $64 \%$ informed the patients of the results. This contrasts with more than $90 \%$ of primary care providers reporting there are benefits to dementia screening including advanced care planning and interventions (1).

This series of papers offers a compelling argument for thinking "outside the box" to increase the likelihood of early detection of MCI-AD and provide potentially actionable methods such as creation of new tools and 
screening platforms, computerized testing, integration of screening tools into electronic health records, and offering in-home testing that does not require any physician or staff time. If the long-term goal is to increase "real world" early MCI and AD detection, diagnosis, and treatment, improve patient care, reduce disparities in health outcomes particularly in underserved communities, and reduce healthcare costs then we must take a transformative approach in our thinking about how best to serve our patients and the communities in which they live. This resonates strongly with the three guiding principles of the National Alzheimer's Project Act (NAPA) (11), especially its third principle: "Transform the way we approach Alzheimer's disease and related dementias."

Funding: This work is supported by grants from the National Institutes of Health (R01 AG040211, R01 NS101483, and R01 AG057681), the Harry T. Mangurian Foundation, and the Leo and Anne Albert Charitable Trust.

Open Access: This article is distributed under the terms of the Creative Commons Attribution 4.0 International License (http://creativecommons.org/ licenses/by/4.0/), which permits use, duplication, adaptation, distribution and reproduction in any medium or format, as long as you give appropriate credit to the original author(s) and the source, provide a link to the Creative Commons license and indicate if changes were made.

\section{References}

1. Alzheimer Association, 2019 Alzheimer's Disease Facts and Figures. https:// alz.org/alzheimers-dementia/ facts-figures. Accessed 2/10/20

2. Galvin JE. Using informant and performance screening methods to detect mild cognitive impairment and dementia. Curr Rep Gerontol 2018; 7:19-25.

3. McKhann GM, Knopman DS, Chertkow H, et al. The diagnosis of dementia due to Alzheimer's disease: recommendations from the National Institute on Aging-Alzheimer's association workgroups on diagnostic guidelines for Alzheimer's disease. Alzheimers Dement. 2011; 7:263-269.

4. Albert MS, DeKosky ST, Dickson D, et al. The diagnosis of mild cognitive impairment due to Alzheimer's disease: recommendations from the National Institute on Aging-Alzheimer's association workgroups on diagnostic guidelines for Alzheimer's disease. Alzheimers Dement. 2011;7:270-279.

5. Tolea MI, Galvin JE. Current Guidelines for Dementia Screening: Shortcomings and Recommended Changes. Neurodegener Dis Manag 2013;3:565-573.

6. Galvin JE. Dementia screening, biomarkers and protein misfolding: Implications for public health and diagnosis. Prion. 2011;5:16-21

7. Sabbagh M, Boada M, Borson S, et al. Rational for early detection of Mild Cognitive Impairment (MCI). J Prev Alz Dis 2020;3(7):158-164

8. Sabbagh M, Boada M, Borson S, et al. Early detection of Mild Cognitive Impairment (MCI) in primary care. J Prev Alz Dis 2020;3(7):165-170

9. Sabbagh M, Boada M, Borson S, et al. Early detection of Mild Cognitive Impairment (MCI) in an at-home setting. J Prev Alz Dis 2020;3(7):171-178

10. Holsinger T, Boustani M, Abbot D, Williams JW. Acceptability of dementia screening in primary care patients. Int J Geriatr Psychiatry. 2011;26:373-379

11. The National Alzheimer's Project Act. https://aspe.hhs.gov/nationalalzheimers-project-act. Accessed 2/10/20. 